Radial and Nonradial Pulsations as Probes of Stellar Physics

ASP Conference Series, Vol. 259, 2002

C. Aerts, T.R. Bedding, \& J. Christensen-Dalsgaard, eds.

\title{
The COROT Mission and its Seismology Programme
}

\author{
A. Baglin, M. Auvergne, C. Catala, E. Michel, M.J. Goupil, R. Samadi, \\ B. Popielsky
}

DESPA, Observatoire de Paris, France

and the COROT Team

\begin{abstract}
The COROT project, developed in the framework of the CNES small satellite programme with a wide European cooperation, will be launched in 2004. It is dedicated to seismology and the detection of telluric planets. It will perform relative broad-band photometry in visible light, during very long (150 days) observing runs in the same direction. Both programmes are working simultaneously on the same region of the sky. The seismology programme aims at mapping the HR diagram and study in detail 50 targets brighter than 9th magnitude. Another 50 will also be observed with a lower accuracy. The exoplanet programme will search for telluric planets slightly larger than the Earth, in the habitable zone and closer. Its data will also address many seismology problems.
\end{abstract}

\section{History and organisation of the project}

COROT is a minisatellite, i.e. a mission of intermediate size and low cost. Its total mass is less than $600 \mathrm{Kg}$. It has a low Earth orbit and a small telemetry rate. Its cost remains below 80 MEuros.

It was proposed first in 1993 by C. Catala, M. Auvergne and A. Baglin, (Catala et al., 1994) in answer to a call for ideas from CNES for "small missions", using the generic CNES platform PROTEUS. It was preselected in 1994, as a pure asteoseismology mission for launch in 1997. The final decision was taken in October 2000 , in a more complex and more promising framework. The launch is planned for 2004. Many European countries have joined the project.

CNES has the global responsibility for the mission. In France, three space laboratories contribute to the hardware, and many more to the scientific preparation. RSSD/ESTEC, Austria, Belgium, Italy, Germany, Spain and the ESA Science Programme contribute.

\section{Instrument and Mission profile}

Very high photometric accuracy is needed for both programmes. The scientific specification in seismology is to be able to detect solar-like oscillations of $2 \mathrm{ppm}$ with a confidence level of $99 \%$, during 5 -day observing runs, on a 6 th magnitude star. The exoplanet programme asks for detection of a transit of a telluric planet 
orbiting around a $\mathrm{G} / \mathrm{K}$ dwarf. This can be obtained with a $600 \mathrm{~cm}^{2}$ telescope, in a broad band centered on the visible, but with very strict control of all the sources of noise which have to remain much smaller than the photon noise (Baglin et al., 1998).

The focal plane is composed of four CCDs, with an optimized quantum efficiency. Two are dedicated to seismology, looking at a few bright targets (10 stars with $m_{v} \leq 9$ ), the two others, dedicated to the exoplanet programme, observe a very large number of faint stars (12000 stars down to $\left.m_{v} \leq 16\right)$. The exoplanet field will be almost on focus while the seismology field is highly defocused to ensure the photometric accuracy.

But the major characteristic feature of COROT, as compared to the other present space projects devoted to high precision stellar photometry, is its possibility of performing very long observations on the same field (up to 150 days). This can be done in a low Earth orbit, but restricts the observable sky; it is composed of two circles in opposite directions, centered on the equatorial plane; their size $(\sim 10$ degrees $)$ is induced by the altitude of the orbit and the level of protection against stray light from the Earth; the position along the equator has been chosen to optimize the scientific quality of the observable targets in both programmes; it is very close to the galactic plane.

\section{The seismology programme}

\subsection{The targets}

As described in Baglin et al. (1998) at least 5 long runs of 150 days' duration are foreseen. For each of them the seismology plane will look at one 6th magnitude star (called the main target) and 9 fainter ones in the field, called secondary targets. The choice of the observable sky allows the observation of more than 10 of such primary target candidates, nicely distributed in the HR diagram as shown on Fig. 1 (left). The corresponding secondary targets are not yet chosen, but an example of a possible coverage of the HR diagram is given on Fig. 1 (right).

An extended programme of ground-based observations is under development to characterise these targets with the highest possible accuracy (Sterken, 2001).

\subsection{Examples of the diagnostic power of the COROT data}

Its long durations of observation give a very high accuracy in the power spectrum, both on amplitudes and frequencies. The quality foreseen for COROT on a 6th magnitude star is equivalent to what was reached with the IPHIR/PHOBOS solar experiment (Fig. 2, left). It will allow us for instance to locate the base of the convective envelope in low mass stars (Monteiro et al., 2000) and more generally the uncertainty on the frequency determination decides the accuracy of the inversion process (Fig. 2 right).

This high frequency accuracy will also allow a precise measurement of line profiles, amplitudes and life-times of the modes. In the case of solar-like stochastically excited oscillations, it will provide powerful diagnostics of the hydrodynamical properties of the very outer convective zone (Samadi et al., 2001), as illustrated in Fig. 3. 

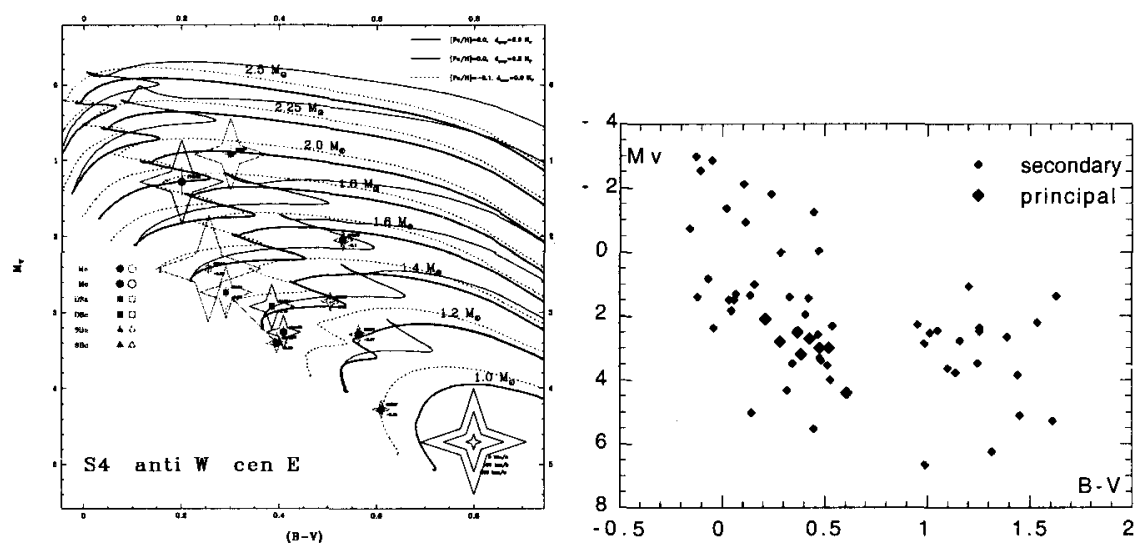

Figure 1. Left: HR diagrams of the possible principal targets (from B. Popilesky). Right: possible choice of secondary targets.
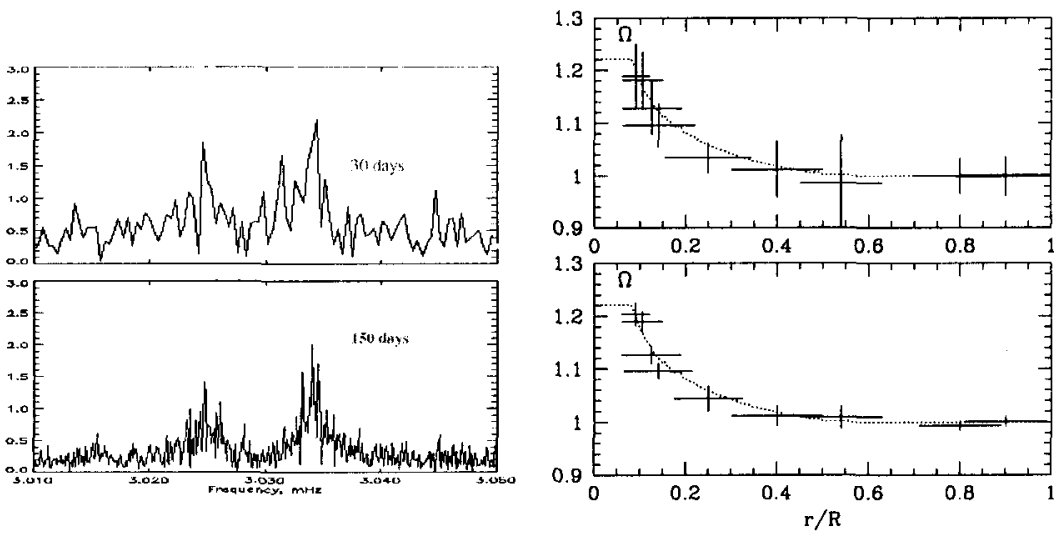

Figure 2. Left: Power spectra of the IPHIR data, for 30 and 150 days (Toutain and Frölich, 1992). Right: Inversion of the rotation profile of an A7 star using frequency accuracies corresponding to these durations.

\subsection{Seismology in the exoplanet field}

Furthermore, the data collected in the exoplanet field will be available to the whole scientific community for adressing other subjects than the exoplanet detection, after an $\mathrm{AO}$ issued by the Scientific Committee. For $m_{v}=15.5$, the noise level at this magnitude is $\sim 710^{-4}$ in 1 hour. Three colours will be available for the brightest objects. In all categories of variable stars known from the ground it will improve the signal-to-noise ratio by a factor of 10 to 100 , and 

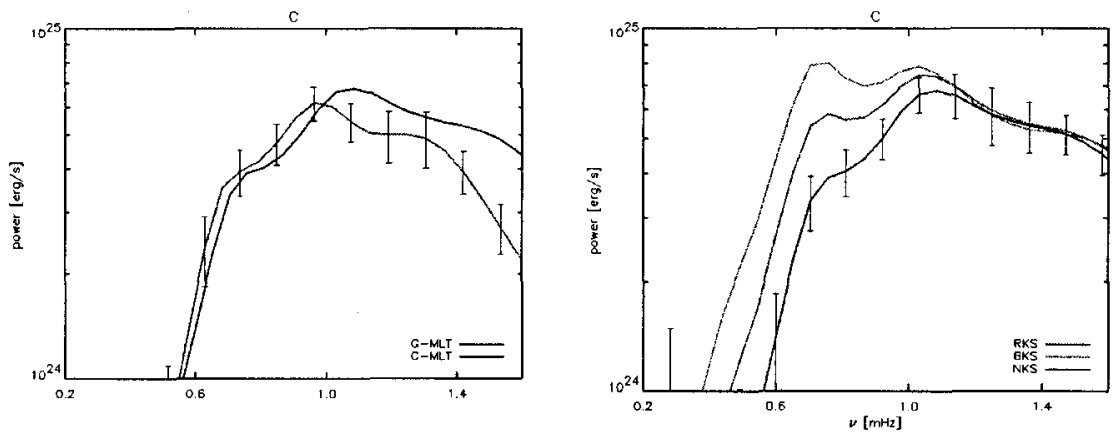

Figure 3. The sensitivity of the shape of the power spectrum to the convective efficiency (left) and to the turbulent spectrum (right), compared to the accuracy reachable with COROT.

certainly detect many more of them. More information on the mission can be found at:

$$
\text { http://corot.astrsp-mrs.fr }
$$

\section{References}

Baglin, A., and the COROT Team 1998, in New eyes to see inside the Sun and stars, eds. F.-L. Deubner, et al. (Kluwer Academic Publishers), 301

Catala, C., Auvergne, M., \& Baglin, A. 1994, ASP Conf. Ser.,Vol. 76, GONG '94: Helio- and Astero-Seismology from the Earth and Space, eds. R.K. Ulrich, E.J. Rhodes Jr., \& W. Dappen, (San Francisco ASP), 426

Toutain, T. \& Fröhlich, C. 1992, A\&A, 257, 287.

Monteiro, M., Christensen-Dalsgaard, J., \& Thompson, M., 2000, MNRAS, 316, 165.

Samadi, R., Goupil, M-J., \& Lebreton, Y. 2001, A\&A, in press.

Sterken, C., ed. 2001, First COROT-MONS-MOST Ground-based SupportWorkshop, (University of Brussels) 